\title{
Global Dynamics of an SEIR Epidemic model with saturated incidence under treatment
}

\author{
M. Badole ${ }^{*}$, S. K. Tiwari ${ }^{2}$, V. Gupta ${ }^{3}$, A. Agrawal $^{4}$ \\ ${ }^{1}$ School of Studies in Mathematics, Vikram University, Ujjain, India \\ ${ }^{2}$ School of Studies in Mathematics, Vikram University, Ujjain, India \\ ${ }^{3}$ Department of Mathematics, Govt. Kalidas Girl's College, Ujjain, India \\ ${ }^{4}$ Department of Mathematics, Acropolis Institute of Technology and Research, Indore, India \\ *Corresponding Author: monikabadole@gmail.com, Tel.: 9826272235
}

Available online at: www.isroset.org

Received: 06/May/2018, Revised: 19/May/2018/2018, Accepted: 13/Jun/2018, Online: 30/Jun/2018

\begin{abstract}
An SEIR epidemic model with saturated incidence rate under a limited resource for treatment function which is proposed by W. Wang (2006) is investigated in this paper. We have assumed that treatment rate is proportional to the number of infective when it is below the capacity and is a constant when the number of infective is larger than the capacity. The existing threshold conditions of all kinds of the equilibrium points are obtained. The local and global stability of the disease free equilibrium and the endemic equilibrium of the model are discussed. The local asymptotical stability of equilibrium is verified by analyzing the eigen values and using the Routh-Hurwitz criterion. We also discuss the global asymptotical stability of the disease free equilibrium by using, Lyapunov function and endemic equilibrium by autonomous convergence theorem. The study indicates that we should improve the efficiency and enlarge the capacity of the treatment to control the spread of disease. Finally, numerical simulations are given to illustrate the validity of the proposed results.
\end{abstract}

Keywords - SEIR epidemic model, Treatment, Basic reproduction number, Lyapunov function, Stability analysis.

2010 subject classification: 92D30, 93A30, 93D30, 34D23.

\section{INTRODUCTION}

Mathematical models describing the population dynamics of infectious diseases have been playing an important role in a better understanding of epidemiological patterns and disease control for a long time. Various epidemic models have been established and investigated extensively, which leads to the huge progress in the studies of disease control and prevention (See, for example [2-6]. In classical epidemic models, it is usually assumed that the recovered rate of the infection is proportional to the number of the infective.

Furthermore, mathematical models now plays a key role in policy making, including health-economic aspects, emergency planning and risk assessment, control-program evaluation, and optimizing various detection. One of the fundamental results in mathematical epidemiology is that most mathematical epidemic models usually exhibit "threshold" behavior stated as follows: if the average number of secondary infections caused by an average infective, called the basic reproduction number, is less than one the disease will die out, while if it exceeds one there will be an endemic. Most of the models in mathematical epidemiology are compartmental models, with the population being divided into compartments with the assumptions about the nature and time rate of transfer from one compartment to another. These models are often referred to as compartmental models since the individuals in the population are divided into classes or compartments depending on their disease status. It has been suggested by several authors that the disease transmission process may have a nonlinear incidence rate. This allows one to include behavior changes and prevent unbounded contact rate $[7,2]$. A particular example of such an incidence rate is given by $\frac{\lambda l^{p}}{1+\alpha l^{q}}$, where $p, q, \alpha$ and $\lambda$ are positive. After studying the cholera epidemic spread in Bari in 1973, Capasso and Serio [14] introduced a saturated incidence rate $\mathrm{g}(\mathrm{I}) \mathrm{S}$ into epidemic models, where $\mathrm{g}(\mathrm{l})$ tends to a saturation level when $\mid$ gets large, i.e. $g(l)=\frac{\beta I}{1+\alpha \mid}$ where $\beta \mid$ measures the infection force of the disease and $\frac{1}{1+\alpha l}$ measures the inhibition effect from the behavioral change of the susceptible individuals when their number increases or from the crowding effect of the infective individuals. This 
incidence rate seems more reasonable than the bilinear incidence rate $\mathrm{g}(\mathrm{I}) \mathrm{S}=\beta \mathrm{IS}$ proposed by Kermack and Mckendrick [16] in 1927 because it includes the behavioral change and crowding effect of the infective individuals and prevents the unboundedness of the contact rate by choosing suitable parameters. In many epidemic models, bilinear incidence rate $\beta S \mathrm{SI}$ is frequently used $[9,11]$.

Many diseases such as measles, severe acute respiratory syndromes (SARS) and so on, however, incubate inside the hosts for a period of time before the hosts become infectious. So the systems that are more general than SIR or SIRS types need to be studied to investigate the role of incubation in disease transmission. We may assume that a susceptible individual first goes through a latent period (and said to become exposed or in class E) after infection before becoming infectious. Thus the resulting models are of SEIR (susceptible-exposed-infectious-recovered) or SEIRS (susceptible-exposed-infectious-recovered-susceptible) types, respectively, depending on whether the acquired immunity is permanent or not. However, every country should have a maximal capacity treatment for diseases. Therefore, it is vital to describe the limited capacity for treatment [7]. The treatment is an important method to decrease the spread of diseases. Wang and Ruan in [18], introduced the following constant treatment function of diseases into an SIR epidemic model,

$T(I)= \begin{cases}r, & I>0 \\ 0 & I=0\end{cases}$

which simulated a limited capacity for treatment. Later, Wang [14] considered the piecewise linear treatment function

$T(I)= \begin{cases}r l, & \text { If } 0 \leq \mathrm{I} \leq \mathrm{I}_{0} \\ \mathrm{kl} \mathrm{l}_{0} & \text { IfI }>\mathrm{I}_{0}\end{cases}$

where $\mathrm{k}$ and $\mathrm{I}_{0}$ are positive constants. This means that the treatment rate is proportional to the number of the infective before the capacity of treatment is reached and takes the maximal capacity $\mathrm{kl}_{0}$, otherwise. This type of treatment function is more realistic because in every hospital, the number of beds is limited and also they have a certain capacity of medicines. In this paper, we shall investigate a model which includes a nonlinear incidence rate $\frac{\beta S I}{1+\alpha l}$ and treatment function which was introduced by Wang [17].

Motivated by the work of Jinhoge Zhang, Jianwen Jia and Xinyu Song [8], Kar and Batabyal[13] in this paper, we are concerned an SEIR epidemic model with the effect of saturated incidence rate and the piecewise linear treatment function. The purpose of this paper is to show that the piecewise linear treatment has significant impact on an SEIR epidemic model.

\section{RELATED WORK}

In the year 2012 [10] L. Zhou et.al worked on an SIR epidemic with treatment function $h^{*}(I)=\frac{\alpha I}{\omega+I}$ after this in year 2014 [8] J. Zhang et.al modified the model [10], with saturated incidence rate $\frac{\beta S I}{1+\alpha S}$ and the same treatment function. Recently in the year 2017 [1] A. Agrawal modified the paper of [8] J. Zang et.al. using a SEIRS epidemic model with same incidence rate and treatment function.

\section{METHODOLOGY}

The Mathematical Model and the Existence of Equilibria

In [10] some examples of the SEIR epidemic model with saturated incidence rate and continually differentiable treatment function with the saturated phenomenon of the limited medical resources was studied as follows:

$$
\begin{aligned}
& S^{\prime}(t)=A-\frac{\beta S I}{1+\alpha l}-d S \\
& E^{\prime}(t)=\frac{\beta S I}{1+\alpha l}-(d+\varepsilon) E \\
& I^{\prime}(t)=\varepsilon E-(d+\mu+v) I-\frac{r l}{1+k l} \\
& R^{\prime}(t)=v l-d R+\frac{r l}{1+k l}
\end{aligned}
$$

Another example is SEIRS epidemic model with saturated incidence rate $\frac{\beta S I}{1+\alpha l}$ and treatment function $T(I)=\frac{\mathrm{rl}}{1+\mathrm{kl}}$ generated by [18] included the recovered compartment $\mathrm{R}(\mathrm{t})$ in $\mathrm{S}^{\prime}(\mathrm{t})$ which was studied as follows:

$$
\begin{aligned}
& S^{\prime}(t)=B-d S-\frac{\beta S I}{1+\alpha l}+v R \\
& E^{\prime}(t)=\frac{\beta S I}{1+\alpha l}-(\varepsilon+d) E \\
& I^{\prime}(t)=\varepsilon E-(\gamma+d) I-\frac{r l}{1+k l} \\
& R^{\prime}(t)=\gamma l-(d+v) R+\frac{r l}{1+k l}
\end{aligned}
$$

Based on the above motivations, in this paper, we further explore an SEIR epidemic model with saturated incidence rate $\frac{\beta S I}{1+\alpha l}$ and continually differentiable function $T(I)=\left\{\begin{array}{ll}r l & \text { if } 0 \leq I \leq I_{0} \\ k & \text { if } I>I_{0}\end{array}\right.$ generated by [17] to characterize the saturation phenomenon of limited medical resources. The model can be described by following system of equations: 
$S^{\prime}(t)=A-\frac{\beta S I}{1+\alpha I}-d S$

$E^{\prime}(t)=\frac{\beta S I}{1+\alpha I}-(d+\varepsilon) E$

$I^{\prime}(t)=\varepsilon E-(d+\mu+v) I-T(l)$

$R^{\prime}(t)=v l-d R+T(l)$

where $S(t), E(t), I(t)$ and $R(t) \geq 0$, and $S(t), E(t), I(t)$ and $R(t)$ denote the numbers of susceptible, exposed but not yet infective, infective and recovered individuals at time t. $A$ is recruitment rate of the population, $\alpha$ is saturation factor that measures the inhibitory effect, $\beta$ is the transmission or contact rate, $d$ is natural death rate of population, $\varepsilon$ is the rate of transformation from incubation period individuals to infective individuals, $\mu$ is the diseases related mortality, $v$ is natural recovery rate of the infective individuals, $r$ is maximal medical resources supplied per unit time, and $k$ is saturation factor that measures the effect of the infected being delayed for treatment. $\beta, d, \varepsilon, \mu, v, r, \alpha$ and $k$ all are positive.

Since the first three equations in (1) are independent of variable $R$, it suffices to consider the following reduced model:

$S^{\prime}(t)=A-\frac{\beta S I}{1+\alpha I}-d S$

$E^{\prime}(t)=\frac{\beta S I}{1+\alpha I}-(d+\varepsilon) E$

$I^{\prime}(t)=\varepsilon E-(d+\mu+v) I-T(I)$

In the next section we study the stability of the model taking two different cases of treatment function.

\section{Equilibrium states and their stability}

Case - I when $T(I)=r l$, if $0 \leq I<I_{0}$ our model reduces to

$S^{\prime}(t)=A-\frac{\beta S I}{1+\alpha I}-d S$

$E^{\prime}(t)=\frac{\beta S I}{1+\alpha I}-(d+\varepsilon) E$

$I^{\prime}(t)=\varepsilon E-(d+\mu+v) I-r l$

It follows from system of equations (3) that

$$
\begin{aligned}
(S+E+I)^{\prime} & =A-d(S+E+I)-(\mu+v) I-r l \\
& \leq A-d(S+E+I)
\end{aligned}
$$

Then the $\limsup _{t \rightarrow \infty}(S+E+I) \leq \frac{A}{d}$. Thus the feasible region for the system (3) is

$\Omega=\left\{(\mathrm{S}, \mathrm{E}, \mathrm{I}) \mid \mathrm{S}+\mathrm{E}+\mathrm{I} \leq \frac{\mathrm{A}}{\mathrm{d}}, \mathrm{S}>0, \mathrm{E} \geq 0, \mathrm{I} \geq 0\right\}$
It is easy to verify that the region $\Omega$ is positively invariant with respect to system of equations (3).

Denote reproduction number

$\mathrm{R}_{0}=\frac{\beta \varepsilon A}{\mathrm{~d}(\varepsilon+\mathrm{d})(\mu+\mathrm{d}+\mathrm{r}+\mathrm{v})}$

The system of equations (3) always be diseases free equilibrium $E^{0}\left(\frac{A}{d}, 0,0\right)$. Next, we will find the conditions of the existence of endemic equilibrium.

An endemic equilibrium always satisfies

$S^{\prime}(t)=A-\frac{\beta S I}{1+\alpha I}-d S=0$

$E^{\prime}(t)=\frac{\beta S I}{1+\alpha I}-(d+\varepsilon) E=0$

$I^{\prime}(t)=\varepsilon E-(d+\mu+v) I-r l=0$

By some simple calculation, we have $S^{*}=\frac{A(1+\alpha l)}{\{\beta I+d(1+\alpha l)\}}$, $E^{*}=\frac{\beta A I}{(d+\varepsilon)\{(\beta+\alpha d) I+d\}}$

$I^{*}=\frac{d}{(\beta+\alpha d)}\left[\frac{\beta \varepsilon A}{d(d+\varepsilon)(d+\mu+r+v)}-1\right]$ or $I^{*}=\frac{d}{(\beta+\alpha d)}\left[R_{0}-1\right]$

Lemma.1 The system (1) has a disease free equilibrium points if $\mathrm{N}=\frac{\mathrm{A}}{\mathrm{d}}$.

Proof. Consider $N(t)=S(t)+l(t)+R(t)$

Then have $\frac{d N}{d t}=A-d N(t)$ Simplify and thus $\lim _{t \rightarrow \infty} N(t)=\frac{A}{d}$.

This implies the conclusion.

\section{The Local Stability Analysis of Equilibria}

In this section we will examine the local stability of the equilibria by analyzing the eigen values of the Jacobian matrices of (3) at the equilibria and using Routh-Hurwitz criterion.

Theorem.1 The diseases free equilibrium $E^{0}$ is locally asymptotically stable when $\mathrm{R}_{0}<1$ and is unstable when $\mathrm{R}_{0}>1$.

Proof. The Jacobian matrix of system (3) at $E^{0}$ is

$J_{1}\left(E_{0}\right)=\left(\begin{array}{ccc}-d & 0 & \frac{-\beta A}{d} \\ 0 & -(\varepsilon+d) & \frac{\beta A}{d} \\ 0 & 0 & -(\mu+d+r+v)\end{array}\right)$ 
The characteristics equation of system (3) at $E^{0}$ is the following form:

$(\lambda+d)\left[\lambda^{2}+(\varepsilon+\mu+2 d+r+v) \lambda+(\varepsilon+d)(\mu+d+r+v)\right.$

$\left.-\frac{\beta \varepsilon A}{d}\right]=0$

Clearly, $\lambda_{1}=-d$ is always negative root of (6) and all other roots of (6) are determined by the following equation:

$\lambda^{2}+(\varepsilon+\mu+2 d+r+v) \lambda+(\varepsilon+d)(\mu+d+r+v)-\frac{\beta \varepsilon A}{d}=0$

which has negative roots, if and only if $(\varepsilon+d)(\mu+d+r+v)-\frac{\beta \varepsilon A}{d}>0$. That is $R_{0}<1$. So the diseases free equilibrium $E_{0}$ is locally asymptotically stable when $R_{0}<1$ and is unstable when $R_{0}>1$.

Theorem.2 If $\mathrm{R}_{0}>1$ then the endemic equilibrium $E^{*}\left(S^{*}, E^{*}, I^{*}\right)$ is locally asymptotically stable

Proof. The Jacobian matrix of system (3) at $E^{*}$ is

$$
J_{1}\left(E_{1}^{*}\right)=\left(\begin{array}{ccc}
-d-\frac{\beta l^{*}}{1+\alpha l^{*}} & 0 & -\frac{\beta S^{*}}{\left(1+\alpha l^{*}\right)^{2}} \\
\frac{\beta l^{*}}{1+\alpha l^{*}} & -(\varepsilon+d) & \frac{\beta S^{*}}{\left(1+\alpha l^{*}\right)^{2}} \\
0 & \varepsilon & -(\mu+d+r+v)
\end{array}\right)
$$

The characteristic equation is given by

$\lambda^{3}+a_{1} \lambda^{2}+a_{2} \lambda+a_{3}=0$

where

$$
\begin{aligned}
a_{1}= & \left(d+\frac{\beta l^{*}}{1+\alpha l^{*}}\right)+\varepsilon+\mu+2 d+r+v \\
a_{2}= & \left(d+\frac{\beta l^{*}}{1+\alpha l^{*}}\right)(\varepsilon+\mu+2 d+r+v)+ \\
& (\varepsilon+d)(\mu+d+r+v)-\frac{\beta \varepsilon S^{*}}{\left(1+\alpha l^{*}\right)^{2}} \\
a_{3}= & \left(d+\frac{\beta l^{*}}{1+\alpha l^{*}}\right)(\varepsilon+d)(\mu+d+r+v)-\frac{\beta \varepsilon d S^{*}}{\left(1+\alpha l^{*}\right)^{2}}
\end{aligned}
$$

By direct calculation we have

$a_{1}>0, a_{2}>0$ if $\frac{\beta \varepsilon S^{*}}{\left(1+\alpha l^{*}\right)^{2}}<0, a_{3}>0$ if $\frac{\beta \varepsilon d S^{*}}{\left(1+\alpha l^{*}\right)^{2}}<0$ and $a_{1} a_{2}-a_{3}>0$. Then by Routh-Hurwitz criterion, it follows that the endemic equilibrium $E^{*}$ is locally asymptotically stable. This completes the proof.

\section{The Global Stability Analysis of Equilibria}

In this section, we analyze the global stability of the diseasefree and endemic steady states. Firstly, we consider the global stability of the disease-free equilibrium.

Theorem.3 If $R_{0}<1$, then the disease-free equilibrium $E^{0}$ is globally asymptotically stable

Proof If $R_{0}<1$ then $R_{0}<1$. From the first equation of (3), we have $\frac{d S}{d t} \leq A-d S$. A solution of the equation $\frac{d y}{d t}=A-d y$ is a maximal solution of $S(t)$. Note that $y \rightarrow \frac{A}{d}$ as $t \rightarrow \infty$. By the comparison theorem, we get $S(t) \leq \frac{A}{d}$, and from the set $\Omega=\left\{(S, E, I) \mid S+E+I \leq \frac{A}{d}, \quad S>0, E \geq 0, I \geq 0\right\} \quad$ we have $\mathrm{l}(\mathrm{t}) \leq \frac{\mathrm{A}}{\mathrm{d}}$

Consider the following Lyapunov function:

$\mathrm{L}=\varepsilon \mathrm{E}+(\mathrm{d}+\varepsilon) \mathrm{I}$

$\mathrm{L}^{\prime}=\varepsilon \mathrm{E}^{\prime}+(\mathrm{d}+\varepsilon) \mathrm{l}^{\prime}$

$L^{\prime}=\left[\frac{\varepsilon \beta S I}{1+\alpha I}-(d+\varepsilon)(\mu+d+r+v) l\right]$

$L^{\prime} \leq\left[\frac{\beta \varepsilon A}{d}-(d+\varepsilon)(\mu+d+r+v)\right]$

$=(d+\varepsilon)(\mu+d+r+v)\left[\frac{\beta \varepsilon A}{d(d+\varepsilon)(\mu+d+r+v)}-1\right]$

$=(d+\varepsilon)(\mu+d+r+v)\left[R_{0}-1\right] \mid \leq 0$

and $L^{\prime}=0$ if and only if $I=0$. The largest compact invariant set in $\left\{(S, E, I) \in \Omega, L^{\prime}=0\right\}$ is the singleton $E^{0}$. Therefore, by Lasalle-Lyapunov theorem, every solution that starts in $\Omega$ approaches $\mathrm{E}^{0}$ as $\mathrm{t} \rightarrow \infty$. This completes the proof.

In the following, we will discuss the global stability of the endemic equilibrium when $\mathrm{R}_{0}<1, \mathrm{k}<\mathrm{k}_{1}$ using the second additive compound matrix. Here we will shortly describe the general method in which the global stability analysis for the endemic equilibrium will be performed through the approach due to $\mathrm{Li}$ and Muldowney [12]. Consider the autonomous dynamical system

$x^{\prime}=f(x)$

where $f: D \rightarrow R^{n}, D \subset R^{n}$ is open set and is simply connected, and $x \in D, x \mapsto f^{\prime \prime}(x) \in R^{n}, f(x) \in C^{1}(D)$.

Let $x^{*}$ be a equilibrium of (11). We recall that $x^{*}$ is said to be globally stable in $D$ if it is locally stable and all trajectories in $D$ converge to ${ }^{*}$. Assume that the following hypotheses hold.

(H1) There exists a compact absorbing set $\mathrm{K} \subset \mathrm{D}$.

$(H 2) \quad$ Equation (11) has a unique equilibrium $\mathrm{x}^{*}$ in $D$. 
The basic idea of this method is that if the equilibrium $x^{*}$ is locally stable, then the stability is assured provided that $(H 1)$ and $(H 2)$ hold and no non-constant periodic solution of (11) exists. Therefore, sufficient conditions on $f$ capable of precluding the existence of such solutions have to be detected. Li and Muldowney showed that if $(H 1)$ and $(H 2)$ hold and (11) satisfy a Bendixson criterion that is robust under $\mathrm{C}^{1}$ local $\epsilon$-perturbations of $f$ at all non-equilibrium non-wandering points for (11), then $\mathrm{x}^{*}$ is globally stable and robust under $C^{1}$ local $\varepsilon$-perturbation. Let $P(x)$ be a $\left(\begin{array}{l}n \\ 2\end{array}\right) \times\left(\begin{array}{l}n \\ 2\end{array}\right)$ matrix valued function, that is, $C^{1}$, on $D$ and consider

$B=P_{f} P^{-1}+P \frac{\partial f^{[2]}}{\partial x} P^{-1}$

where $P_{f}$ is

$\frac{\partial \mathrm{P}_{\mathrm{ij}}^{*}}{\partial \mathbf{x}} \mathbf{f}=\left.\frac{\partial \mathrm{P}_{\mathrm{ij}}}{\partial \mathrm{t}}\right|_{(11)}$

and the matrix $J^{[2]}$ is the second additive compound matrix of the Jacobian matrix $J$, that is, $J(x)=D f(x)$. Generally speaking, for an $n \times n$ matrix $J=\left(J_{i j}\right), J^{[2]}$ is a $\left(\begin{array}{l}n \\ 2\end{array}\right) \times\left(\begin{array}{l}n \\ 2\end{array}\right)$ matrix and in the special case $\mathrm{n}=3$ one has

$J^{[2]}=\left(\begin{array}{ccc}J_{11}+J_{22} & J_{23} & -J_{13} \\ J_{32} & J_{11}+J_{33} & J_{12} \\ -J_{31} & J_{21} & J_{22}+J_{33}\end{array}\right)$

Consider the Lozinskil measure $\mu$ of $\mathrm{B}$ with respect to a vector norm $\|\cdot\|$ in $\mathrm{R}^{\mathrm{n}}, \mathrm{N}=\left(\begin{array}{l}\mathrm{n} \\ 2\end{array}\right)$ defined by

$\mu(B)=\lim _{h \rightarrow 0^{+}} \frac{\|l+h B\|-1}{h}$

It is proved in [12] that if $(H 1)$ and $(H 2)$ hold, condition

$$
q=\operatorname{limsupsup}_{t \rightarrow \infty} \frac{1}{x_{0} \in k} \int_{0}^{t} \mu\left(B\left(x\left(s, x_{0}\right)\right)\right) d s<0
$$

It is shown in [12] that there are no orbits giving rise to a simple closed rectifiable curve in $D$ which is invariant for (11), that is, periodic orbits, homoclinic orbits, and heteroclinic cycles. In particular, condition (16) is proved to be a robust Bendixson criterion for (11). Besides, it is remarked that, under assumptions $(H 1)$ and $(H 2)$, condition (16) also implies the local stability of $x^{*}$.

The analysis of the global stability of the endemic equilibrium may be usefully approached by means of the Poincare- Bendixson trichotomy. If the endemic equilibriums globally asymptotically stable, then the disease will permanently be present in the population in case of infinitesimal initial prevalence. Here we will provide an analytical proof of global stability of $\mathrm{E}^{*}$ by giving sufficient conditions. Global stability analysis for the endemic equilibrium will be perform through the approach due to $\mathrm{Li}$ and Muldowney. The instability of $E^{0}$ implies the uniform persistence; that is, there exists a constant $a>0$ such that any solution $(S(t), E(t), I(t))$ with $(S(0), E(0), I(0))$ in the orbit of the system satisfies

$\min \left\{\liminf _{t \rightarrow \infty} S(t), \liminf _{t \rightarrow \infty} E(t), \liminf _{t \rightarrow \infty} S(t)\right\}>a$

Lemma.2 Assume that D is simply connected and that the assumptions (H1) and (H2) hold. Then the unique equilibrium $x^{*}$ of (11) is globally stable in $D$ if $q<0$.

Now, we study the global stability of the endemic equilibrium $\mathrm{P}^{*}$ and obtain.

Theorem.4 If $\mathrm{R}_{0}>1$ then the endemic equilibrium $\mathrm{P}^{*}$ of the system (31) is globally stable.

Proof: The Jacobian matrix of system (3) is

$$
J_{1}\left(E_{2}^{*}\right)=\left(\begin{array}{ccc}
-d-\frac{\beta I}{1+\alpha l} & 0 & -\frac{\beta S}{(1+\alpha l)^{2}} \\
\frac{\beta I}{1+\alpha l} & -(\varepsilon+d) & \frac{\beta S}{(1+\alpha l)^{2}} \\
0 & \varepsilon & -(\mu+d+r+v)
\end{array}\right)
$$

and its second additive compound matrix is

$$
\mathrm{J}_{1}^{[2]}=\left(\begin{array}{crcc}
-(\varepsilon+2 d)-\frac{\beta I}{1+\alpha l} & \frac{\beta S}{(1+\alpha l)^{2}} & \frac{\beta S}{(1+\alpha l)^{2}} \\
\varepsilon & -\frac{\beta I}{(1+\alpha l)}-(\mu+2 d+r+v) & 0 \\
0 & \frac{\beta I}{(1+\alpha l)} & -(\varepsilon+\mu+2 d \\
& & +r+v)
\end{array}\right)
$$

Choose the function $P=P(S, E, I)=\operatorname{diag}\left(1, \frac{E}{I}, \frac{E}{I}\right)$; it follows that

$P^{-1}=\operatorname{diag}\left(1, \frac{I}{E}, \frac{I}{E}\right)$ and $P_{f}=\operatorname{diag}\left(0, \frac{E^{\prime} I-I^{\prime} E}{I^{2}}, \frac{E^{\prime} I-I^{\prime} E}{I^{2}}\right)$

Also we have

$$
P_{f} P^{-1}=\operatorname{diag}\left(0, \frac{E^{\prime}}{E}-\frac{I^{\prime}}{l}, \frac{E^{\prime}}{E}-\frac{I^{\prime}}{l}\right)
$$




$$
P J_{1}^{[2]} P^{-1}=\left(\begin{array}{cccc}
-(\varepsilon+2 d)-\frac{\beta I}{1+\alpha l} & \frac{\beta S I}{(1+\alpha l)^{2} E} & \frac{\beta S I}{(1+\alpha l)^{2} E} \\
\frac{\varepsilon E}{l} & -\frac{\beta I}{(1+\alpha l)} & -(\mu+2 d+r+v) & 0 \\
0 & \frac{\beta I}{(1+\alpha l)} & -\left(\begin{array}{l}
\varepsilon+\mu+2 d \\
+r+v
\end{array}\right)
\end{array}\right)
$$

The matrix $B=P_{f} P^{-1}+P^{[2]} P^{-1}$ can be written in the matrix form

$$
B=\left(\begin{array}{ll}
B_{11} & B_{12} \\
B_{21} & B_{22}
\end{array}\right)
$$

where

$$
\begin{aligned}
& \mathrm{B}_{11}=-(\varepsilon+2 d)-\frac{\beta I}{1+\alpha l} \\
& \mathrm{~B}_{12}=\left(\frac{\beta S I}{(1+\alpha l)^{2} \mathrm{E}}, \frac{\beta S I}{(1+\alpha l)^{2} \mathrm{E}}\right) \\
& \mathrm{B}_{21}=\left(\frac{\varepsilon \mathrm{E}}{\mathrm{I}}, 0\right) \\
& \mathrm{B}_{22}=\left(\begin{array}{cc}
-\frac{\beta \mathrm{I}}{1+\alpha I}-(\mu+2 d+r+v)+\frac{\mathrm{E}^{\prime}}{\mathrm{E}}-\frac{\mathrm{I}^{\prime}}{\mathrm{I}} & 0 \\
\frac{\beta I}{1+\alpha l} & -(\varepsilon+\mu+2 d+r+v)+\frac{\mathrm{E}^{\prime}}{\mathrm{E}}-\frac{\mathrm{I}^{\prime}}{\mathrm{I}}
\end{array}\right)
\end{aligned}
$$

Let $(u, v, w)$ be vector in $R^{3}$; its norm $\|\cdot\|$ is defined as

$\|(u, v, w)\|=\max \{|u|,|v|, \mid w\} \mid$

Let $\mu(B)$ be the Lozinski I measure with respect to this norm.

We choose

$\mu(B) \leq \sup \left\{g_{1}, g_{2}\right\}$

where $g_{1}=\mu_{1}\left(B_{11}\right)+\left|B_{12}\right|, \quad g_{2}=\mu_{1}\left(B_{22}\right)+\left|B_{21}\right|, \quad\left|B_{12}\right|$ and $\left|B_{21}\right|$ are matrix norm with respect to $I_{1}$ vector norm and $\mu_{1}$ denotes the Lozinski I measure with respect to $I_{1}$ norm, then

$\mu_{1}\left(B_{11}\right)={ }_{1}-(\varepsilon+2 d)-\frac{\beta I}{1+\alpha}$

$\left|\mathrm{B}_{12}\right|=\frac{\beta \mathrm{SI}}{(1+\alpha \mid)^{2} \mathrm{E}}$ and $\left|\mathrm{B}_{21}\right|=\frac{\varepsilon \mathrm{E}}{\mathrm{I}}$

Now calculating $\mu_{1}\left(B_{22}\right)$, taking the non diagonal elements of each column of $B_{22}$ in absolute value, and then adding to the corresponding columns of the diagonal elements, we get

$B_{22}^{\prime}=\left(\begin{array}{ccc}-(\mu+2 d+r+v)+\frac{E^{\prime}}{E}-\frac{l^{\prime}}{l} & 0 \\ \frac{\beta I}{1+\alpha l} & -(\varepsilon+\mu+2 d+r+v)+\frac{E^{\prime}}{E}-\frac{I^{\prime}}{I}\end{array}\right)$

Take a maximum of two diagonal elements of $B_{22}^{\prime}$; we have

$$
\begin{aligned}
\mu_{1}\left(B_{22}\right)= & \max \left\{-(\mu+2 d+r+v)+\frac{E^{\prime}}{E}-\frac{l^{\prime}}{I^{\prime}},-(\varepsilon+\mu+2 d+r+v)+\frac{E^{\prime}}{E}-\frac{I^{\prime}}{I^{\prime}}\right\} \\
& =-(\mu+2 d+r+v)+\frac{E^{\prime}}{E}-\frac{l^{\prime}}{l}
\end{aligned}
$$

Therefore we have

$\left.\begin{array}{l}g_{1}=\mu_{1}\left(B_{11}\right)+\left|B_{12}\right|=\frac{\beta S I}{(1+\alpha)^{2} E}-(\varepsilon+2 d)-\frac{\beta I}{1+\alpha l} \\ g_{2}=\mu_{1}\left(B_{22}\right)+\left|B_{21}\right|=-(\mu+2 d+r+v)+\frac{E^{\prime}}{E}-\frac{I^{\prime}}{l}+\frac{\varepsilon E}{I}\end{array}\right\}$

From (1), we have

$\frac{E^{\prime}}{E}=\frac{\beta S I}{(1+\alpha l) E}-(d+\varepsilon)$ and $\frac{I^{\prime}}{l}=\frac{\varepsilon E}{I}-(\mu+d+r+v)$

Then we have

$g_{1}=-(\varepsilon+2 d)-\frac{\beta I}{(1+\alpha l)}+\frac{\beta S I}{(1+\alpha l)^{2} E} \leq \frac{\beta S I}{(1+\alpha l) E}-(\varepsilon+2 d)$

$g_{2}=\frac{\varepsilon E}{l}-(\mu+2 d+r+v)+\frac{E^{\prime}}{E}-\frac{l^{\prime}}{l}=\frac{\varepsilon E}{I}-(\mu+d+r+v)-d+\frac{E^{\prime}}{E}-\frac{I^{\prime}}{I}$

Then from equation (28)

$g_{1} \leq \frac{E^{\prime}}{E}-d$ and $g_{2}=\frac{E^{\prime}}{E}-d \leq \frac{E^{\prime}}{E}-(d-r)$

Furthermore, we obtain

$\mu(B) \leq \sup \left\{g_{1}, g_{2}\right\}$

$\leq\left\{\frac{E^{\prime}}{E}-d, \frac{E^{\prime}}{E}-(d-r)\right\}$

$\leq \frac{E^{\prime}}{E}-(d-r)$

By integrating both sides at the same time, we obtain

$\frac{1}{\mathrm{t}} \int_{0}^{\mathrm{t}} \mu(\mathrm{B}) \mathrm{ds} \leq \frac{1}{\mathrm{t}} \ln \frac{\mathrm{E}(\mathrm{t})}{\mathrm{E}(0)}-(\mathrm{d}-\mathrm{r})$,

$\operatorname{limsupsup}_{t \rightarrow \infty} \frac{1}{t} \int_{0}^{t} \mu(B) d s<-(d-r)<0$

Thus, by the result of [12] it implies that $P^{*}$ is globally asymptotically stable.

\section{Equilibrium states and their stability}

Case - II when $T(I)=r l$, ifl $>I_{0}$

Equilibrium states and their stability

In this case our model reduces to

$S^{\prime}(t)=A-\frac{\beta S I}{1+\alpha I}-d S$

$E^{\prime}(t)=\frac{\beta S I}{1+\alpha l}-(d+\varepsilon) E$

$I^{\prime}(t)=\varepsilon E-(d+\mu+v) I-k$ 
It follows from system of equations (32) that

$$
\begin{aligned}
(S+E+I)^{\prime} & =A-d(S+E+I)-(\mu+v) I-k \\
& \leq A-d(S+E+I)
\end{aligned}
$$

Then the $\limsup _{t \rightarrow \infty}(S+E+I) \leq \frac{A}{d}$

The Local stability analysis of endemic equilibrium points In this section we will examine the local stability of the endemic equilibrium point $E^{*}\left(S^{*}, E^{*}, I^{*}\right)$ by analyzing the eigen values of the Jacobian matrices of equation (31) at the endemic equilibrium points and using Routh-Hurwitz criterion.

Theorem.5 If $\mathrm{R}_{0}>1$ then the endemic equilibrium $E^{*}\left(S^{*}, E^{*}, I^{*}\right)$ is locally asymptotically stable.

Proof. The Jacobian matrix of system (3) at $E^{*}$ is

$$
J_{2}\left(E_{1}^{*}\right)=\left(\begin{array}{ccc}
-d-\frac{\beta I^{*}}{1+\alpha l^{*}} & 0 & -\frac{\beta S^{*}}{\left(1+\alpha l^{*}\right)^{2}} \\
\frac{\beta l^{*}}{1+\alpha l^{*}} & -(\varepsilon+d) & \frac{\beta S^{*}}{\left(1+\alpha l^{*}\right)^{2}} \\
0 & \varepsilon & -(\mu+d+v)
\end{array}\right)
$$

The characteristic equation is given by

$\lambda^{3}+b_{1} \lambda^{2}+b_{2} \lambda+b_{3}=0$

where

$$
\begin{aligned}
b_{1}= & \left(d+\frac{\beta l^{*}}{1+\alpha l^{*}}\right)+\varepsilon+\mu+2 d+v>0 \\
b_{2}= & \left(d+\frac{\beta l^{*}}{1+\alpha l^{*}}\right)(\varepsilon+\mu+2 d+v)+(\varepsilon+d)(\mu+d+v) \\
& -\frac{\beta \varepsilon S^{*}}{\left(1+\alpha l^{*}\right)^{2}} \\
b_{3}= & \left(d+\frac{\beta l^{*}}{1+\alpha l^{*}}\right)(\varepsilon+d)(\mu+d+v)-\frac{\beta \varepsilon d S^{*}}{\left(1+\alpha l^{*}\right)^{2}}
\end{aligned}
$$

By direct calculation we have

$b_{1}>0, b_{2}>0$ if $\frac{\beta \varepsilon S^{*}}{\left(1+\alpha l^{*}\right)^{2}}<0, b_{3}>0$ if $\frac{\beta \varepsilon d S^{*}}{\left(1+\alpha l^{*}\right)^{2}}<0$ and $b_{1} b_{2}-b_{3}>0$. Then by Routh-Hurwitz criterion, it follows that the endemic equilibrium $E^{*}$ is locally asymptotically stable. This completes the proof.

The Global stability analysis of endemic equilibrium points

In this section, we analyze the global stability of the endemic steady states.

Theorem.6 If $R_{0}>1$ then the endemic equilibrium $P^{*}$ of the system (32) is globally stable
Proof: The Jacobian matrix of system (32) is

$$
J_{2}\left(E_{2}^{*}\right)=\left(\begin{array}{ccc}
-d-\frac{\beta I}{1+\alpha l} & 0 & -\frac{\beta S}{(1+\alpha l)^{2}} \\
\frac{\beta I}{1+\alpha l} & -(\varepsilon+d) & \frac{\beta S}{(1+\alpha l)^{2}} \\
0 & \varepsilon & -(\mu+d+v)
\end{array}\right)
$$

and its second additive compound matrix is

$$
\mathrm{J}_{2}^{[2]}=\left(\begin{array}{ccc}
-(\varepsilon+2 \mathrm{~d})-\frac{\beta \mathrm{I}}{1+\alpha \mathrm{l}} \frac{\beta S}{(1+\alpha l)^{2}} & \frac{\beta S}{(1+\alpha l)^{2}} \\
\varepsilon & -\frac{\beta \mathrm{I}}{(1+\alpha \mathrm{l})}-(\mu+2 \mathrm{~d}+\mathrm{v}) & 0 \\
0 & \frac{\beta \mathrm{I}}{(1+\alpha \mathrm{l})} & -(\varepsilon+\mu+2 \mathrm{~d}+\mathrm{v})
\end{array}\right)
$$

Choose the function $Q=Q(S, E, I)=\operatorname{diag}\left(1, \frac{E}{I}, \frac{E}{l}\right)$; it follows that

$Q^{-1}=\operatorname{diag}\left(1, \frac{I}{E}, \frac{I}{E}\right)$ and $Q_{f}=\operatorname{diag}\left(0, \frac{E^{\prime} I-I^{\prime} E}{l^{2}}, \frac{E^{\prime} I-I^{\prime} E}{l^{2}}\right)$

Also we have

$$
\begin{aligned}
& Q_{f} Q^{-1}=\operatorname{diag}\left(0, \frac{E^{\prime}}{E}-\frac{I^{\prime}}{I}, \frac{E^{\prime}}{E}-\frac{I^{\prime}}{l}\right) \\
& Q^{J^{[2]}} Q^{-1}=\left(\begin{array}{ccc}
-(\varepsilon+2 d)-\frac{\beta I}{1+\alpha l} \frac{\beta S I}{(1+\alpha l)^{2} E} & \frac{\beta S I}{(1+\alpha l)^{2} E} \\
\frac{\varepsilon E}{l} & -\frac{\beta I}{(1+\alpha l)}-(\mu+2 d+v) & 0 \\
0 & \frac{\beta I}{(1+\alpha l)} & -\left(\begin{array}{c}
\varepsilon+\mu+ \\
2 d+v
\end{array}\right)
\end{array}\right)
\end{aligned}
$$

The matrix $C=Q_{f} Q^{-1}+Q^{[2]} Q^{-1}$ can be written in the matrix form

$$
C=\left(\begin{array}{ll}
C_{11} & C_{12} \\
C_{21} & C_{22}
\end{array}\right)
$$

where

$$
\begin{aligned}
& C_{11}=-(\varepsilon+2 d)-\frac{\beta I}{1+\alpha I} \\
& C_{12}=\left(\frac{\beta S I}{(1+\alpha I)^{2} E}, \frac{\beta S I}{(1+\alpha l)^{2} E}\right) \\
& C_{21}=\left(\frac{\varepsilon E}{I}, 0\right) \\
& C_{22}=\left(\begin{array}{cc}
-\frac{\beta I}{1+\alpha l}-(\mu+2 d+v)+\frac{E^{\prime}}{E}-\frac{I^{\prime}}{I} & 0 \\
\frac{\beta I}{1+\alpha l} & -(\varepsilon+\mu+2 d+v)+\frac{E^{\prime}}{E}-\frac{I^{\prime}}{I}
\end{array}\right)
\end{aligned}
$$


Let $(u, v, w)$ be vector in $R^{3}$; its norm $\|\cdot\|$ is defined as

$\|(u, v, w)\|=\max \{|u|,|v|, \mid w\} \mid$

Let $\mu(C)$ be the Lozinskil measure with respect to this norm.

We choose

$\mu(C) \leq \sup \left\{f_{1}, f_{2}\right\}$

where $f_{1}=\mu_{1}\left(C_{11}\right)+\left|C_{12}\right|, \quad f_{2}=\mu_{1}\left(C_{22}\right)+\left|C_{21}\right|, \quad\left|C_{12}\right|$ and $\left|C_{21}\right|$ are matrix norm with respect to $I_{1}$ vector norm and $\mu_{1}$ denotes the Lozinskil measure with respect to $I_{1}$ norm, then

$\mu_{1}\left(C_{11}\right)={ }_{1}-(\varepsilon+2 d)-\frac{\beta I}{1+\alpha l}$

$\left|C_{12}\right|=\frac{\beta S I}{(1+\alpha l)^{2} E}$ and $\left|C_{21}\right|=\frac{\varepsilon E}{I}$

Now calculating $\mu_{1}\left(C_{22}\right)$, taking the non diagonal elements of each column of $\mathrm{C}_{22}$ in absolute value, and then adding to the corresponding columns of the diagonal elements, we get

$C_{22}^{\prime}=\left(\begin{array}{cc}-(\mu+2 d+v)+\frac{E^{\prime}}{E}-\frac{I^{\prime}}{I} & 0 \\ \frac{\beta I}{1+\alpha l} & -(\varepsilon+\mu+2 d+v)+\frac{E^{\prime}}{E}-\frac{I^{\prime}}{I}\end{array}\right)$

Take a maximum of two diagonal elements of $C_{22}^{\prime}$; we have

$$
\begin{aligned}
\mu_{1}\left(C_{22}\right) & =\max \left\{-(\mu+2 d+v)+\frac{E^{\prime}}{E}-\frac{I^{\prime}}{I^{\prime}},-(\varepsilon+\mu+2 d+v)+\frac{E^{\prime}}{E}-\frac{I^{\prime}}{I^{\prime}}\right\} \\
& =-(\mu+2 d+v)+\frac{E^{\prime}}{E}-\frac{I^{\prime}}{I}
\end{aligned}
$$

Therefore we have

$$
\left.\begin{array}{l}
\mathrm{f}_{1}=\mu_{1}\left(\mathrm{C}_{11}\right)+\left|\mathrm{C}_{12}\right|=\frac{\beta S \mathrm{I}}{(1+\alpha \mathrm{l})^{2} \mathrm{E}}-(\varepsilon+2 \mathrm{~d})-\frac{\beta \mathrm{I}}{1+\alpha \mathrm{I}} \\
\mathrm{f}_{2}=\mu_{1}\left(\mathrm{C}_{22}\right)+\left|\mathrm{C}_{21}\right|=-(\mu+2 \mathrm{~d}+\mathrm{v})+\frac{\mathrm{E}^{\prime}}{\mathrm{E}}-\frac{\mathrm{I}^{\prime}}{\mathrm{I}}+\frac{\varepsilon \mathrm{E}}{\mathrm{I}}
\end{array}\right\}
$$

From model (1), we have

$$
\frac{E^{\prime}}{E}=\frac{\beta S I}{(1+\alpha l) E}-(d+\varepsilon) \text { and } \frac{l^{\prime}}{l}=\frac{\varepsilon E}{l}-(\mu+d+v)-\frac{k}{l}
$$

Then we have

$$
\begin{aligned}
\mathrm{f}_{1} & =-(\varepsilon+2 d)-\frac{\beta I}{(1+\alpha l)}+\frac{\beta S I}{(1+\alpha l)^{2} E} \leq \frac{\beta S I}{(1+\alpha l) E}-(\varepsilon+2 d) \\
f_{2} & =\frac{\varepsilon E}{l}-(\mu+2 d+v)+\frac{E^{\prime}}{E}-\frac{l^{\prime}}{l}=\frac{\varepsilon E}{l}-(\mu+d+v)-d+\frac{E^{\prime}}{E}-\frac{I^{\prime}}{I} \\
f_{2} & =\frac{E^{\prime}}{E}-d+\frac{k}{l} \\
& \leq \frac{E^{\prime}}{E}-d
\end{aligned}
$$

Then from equation (46) $f_{1} \leq \frac{E^{\prime}}{E}-d$

$f_{2}=\frac{E^{\prime}}{E}-d+\frac{k}{l} \leq \frac{E^{\prime}}{E}-d \leq \frac{E^{\prime}}{E}-(d-r)$

Furthermore, we obtain

$\mu(C) \leq \sup \left\{f_{1}, f_{2}\right\}$

$\leq\left\{\frac{E^{\prime}}{E}-d, \frac{E^{\prime}}{E}-(d-r)\right\}$

$\leq \frac{E^{\prime}}{E}-(d-r)$

By integrating both sides at the same time, we obtain

$\frac{1}{t} \int_{0}^{t} \mu(C) d s \leq \frac{1}{t} \ln \frac{E(t)}{E(0)}-(d-r)$

$\operatorname{limsupsup}_{t \rightarrow \infty} \frac{1}{t} \int_{0}^{t} \mu(C) d s<-(d-r)<0$

Thus, by the result of [12] it implies that $\mathrm{P}^{*}$ is globally asymptotically stable.

\section{RESULTS AND DISCUSSION}

To see the dynamical behavior of system (1) we will give some numerical simulations. We consider the hypothetical set of parameter values as the following.

Case-I when $0 \leq \mathrm{I} \leq \mathrm{I}_{0}$ :

If we choose the parameters as follows

$A=10 ; d=0.2 ; \beta=0.05 ; \varepsilon=1.2 ; \mu=0.4 ; v=0.2 ; r=2.5$;

$\alpha=1.25$, then we get the unique positive equilibrium point. Here the basic reproduction number $R_{0}=0.6493506<1$, $S(t)$ approaches to its steady state value while $E(t), I(t)$ and $\mathrm{R}(\mathrm{t})$ approach zero as time goes to infinity, the diseases dies out (see in Fig.1)

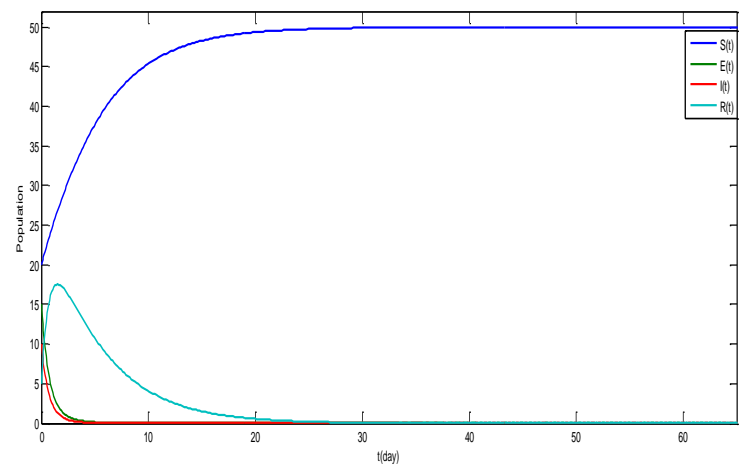

Fig. 1 The figure represents that the diseases dies out

Case-II when $0 \leq \mathrm{I} \leq \mathrm{I}_{0}$ If we choose the parameters as follows: 
$A=10 ; d=0.5 ; \beta=0.3 ; \varepsilon=1.2 ; \mu=0.4 ; v=0.2 ; r=0.1$;

$\alpha=0.8$ then the basic reproduction number $R_{0}=3.52941176>1$. For the above choice of parameters we see that all the four component $S(t) E(t), l(t)$ and $\mathrm{R}(\mathrm{t})$ approach to their steady state values as time goes to infinity, the diseases becomes endemic (see in Fig.2)

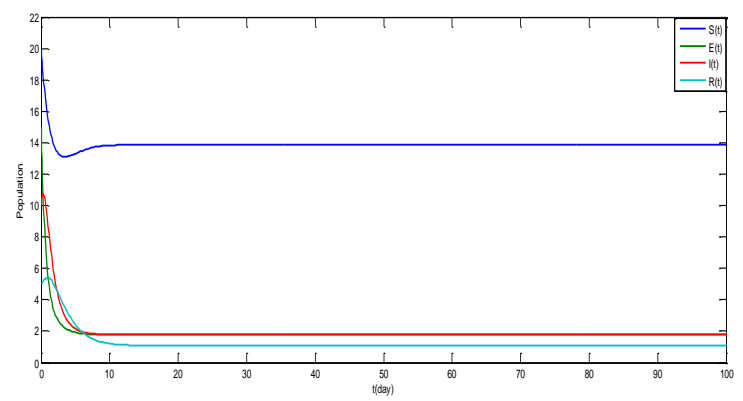

Fig.2The figure represents that the diseases dies endemic Case-III When $I>I_{0}$ : To study the system (32) we choose our parameters as:

$\mathrm{A}=10 ; \mathrm{d}=0.5 ; \mathrm{b}=0.3 ; \varepsilon=1.2 ; \mu=0.4 ; \mathrm{v}=0.2 ; \mathrm{k}=1.25$; $\alpha=1.25$ this shows the dependence of the steady state value of $l^{*}$ of $l(t)$ on the parameter $k$ and we see that $I^{*}$ decreases as $k$ increases. (See Fig 3)

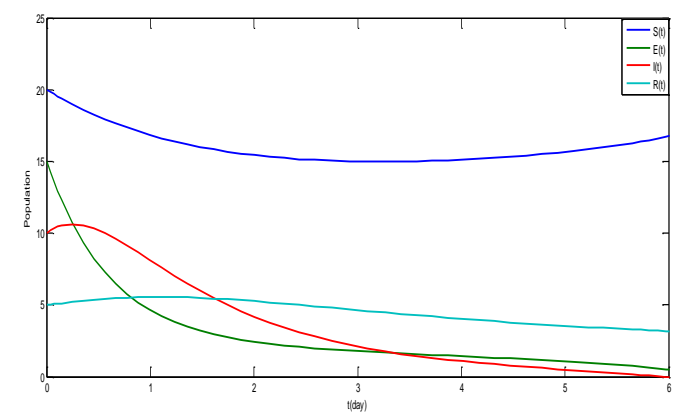

Fig. 3 The figure represents the diseases endemic

Case-IV For value of $r$ $r=0.2, r=0.4, r=0.4$ and $r=1.5$ see in Fig 4 that the dependence of $I^{*}$ on the parameter $r$ and see that $I^{*}$ decreases as $r$ increases.

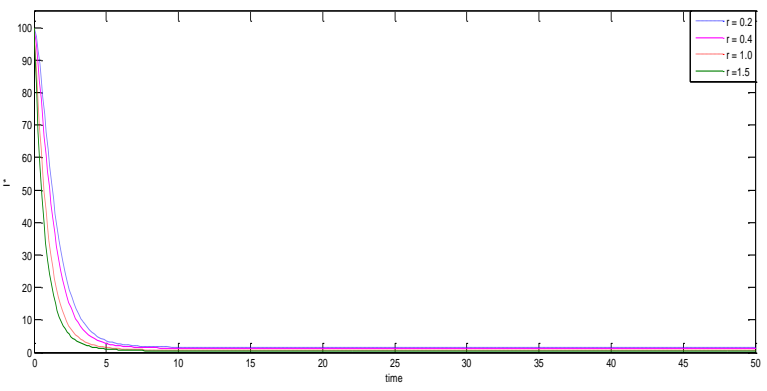

Fig. 4 The figure represents the dependence of $I^{*}$ on the parameter $r$
Case-V In our model parameter $\alpha$ describes the psychological or inhibitory effect. We see Fig5 this shows that the dependence of $I^{*}$ on the parameter $\alpha$, the steady state value $I^{*}$ of the infective decreases as $\alpha$ increases.

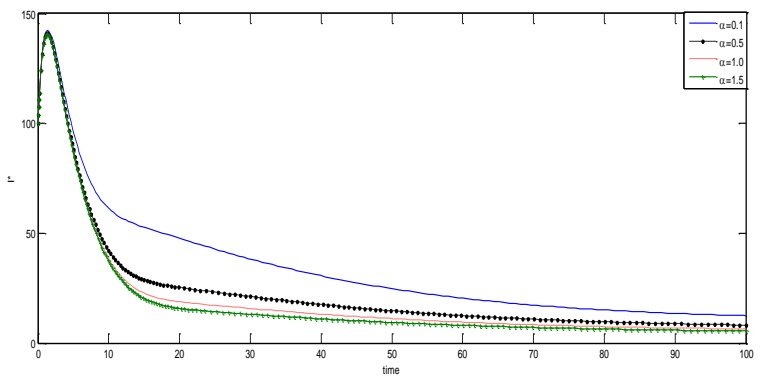

Fig. 5 The figure represents the dependence of $I^{*}$ on the parameter $\alpha$

\section{Conclusion and Future Scope}

In this paper, we consider the SEIR epidemic model with saturated incidence and treatment function which is to understand the effect of delayed treatment on the disease transmission, we have deal with an epidemic model with nonlinear incidence to simulate the limited resources for the treatment of patients, which can occur because patients have to be hospitalized but there are limited beds in hospitals, or there is not enough medicine for treatments. In terms of basic reproduction number the disease-free and endemic equilibrium are discussed. On the basis of stability theory of differential equation, we get some relatively complex conclusion. These results provide useful guidelines to policymaker for public health and can also estimate the impact of control measures for further efforts like patients treatment timely by improving our medical technology and investing more medicines, beds and so on required for eradicate the disease.

\section{REFERENCES}

[1] A. Agrawal, "Global Analysis of an SEIRS epidemic model with saturated incidence and saturated treatment", Asian Journal of Mathematics and Computer Research, Vol. 22(2), pp 43-56, 2017.

[2] D. Xiao, S. Runa, "Global analysis of an epidemic model with non-monotonic incidence rate".Mathematical Bioscience, Vol. 208: pp419-429, 2007.

[3] H.W. Hethcote, "Qualitative analyses of communicable disease models", Mathematical Bioscience, Vol. 28, pp 335-356, 1976.

[4] H.W. Hethcote, P. van den Driessche "Some epidemiological models with nonlinear incidence”, J. Math. Biol. Vol. 29 pp 271-287, 1991.

[5] H.W. Hethcote, "Qualitative analyses of communicable disease models", Mathematical Bioscience, Vol. 28, pp 335-356, 1976.

[6] H.W. Hethcote, "The mathematics of infectious diseases",SIAM Rev Vol. 42, pp 599-653, 2000. 
[7] J. Li, N. Cui. "Dynamic behavior for an SIRS model with nonlinear incidence rate and treatment" Scientific World Journal, Vol 2013, pp 1-5, 2013

[8] J. Zhang, J. Jia and X, Song, "Analysis of an SEIR epidemic model with saturated incidence and saturated treatment function", The Scientific World Journal Vol. 2014, pp1-11, 2014.

[9] L. Acedo, Parr Gonzalez, and A. Arenas, "An exact global solution for the classical epidemic model", Nonlinear Analysis Real World Applications, Vol. 11, no. 3, pp. 1819-1825, 2010.

[10] L. Zhou, M. Fan, "Dynamics of an SIR epidemic model with limited medical resources revisited," Nonlinear Analysis: Real World Applications, Vol. 13, no. 1, pp. 312-324, 2012.

[11] M.E. Alexander and S.M. Moghadas "Bifurcation analysis of SIRS epidemic model with generalized incidence," SIAM Journal on Applied Mathematics, Vol. 65, no. 5, pp. 1794-1816, 2005.

[12] M.Y. Li , J.S. Muldowney, "A geometric approach to global stability problem”, SIAM Journal on Mathematical Analysis, Vol. 27(4), pp 1070-1083, 1996.

[13] T. K .Kar, Batabyal, Ashim., "Modeling and analysis of an epidemic model with non-monotonic incidence rate under treatment", Journal of Mathematics Research, Vol. 2(1), pp103-115, 2010.

[14] V. Capasso, G. Serio, "A generalization of the kermackmckendrick deterministic epidemic mode"l, Math Bioscience Vol. 42, pp 43-61, 1978.

[15] W.M. Liu, S.A. Levin, Y. "I was a, Influence of nonlinear incidence rates upon the behavior of SIRS epidemiological models", J. Math. Biol. Vol. 23, pp 187-204, 1986.

[16] W.O Kermark , A.G. Mckendrick " Contributions to the mathematical theory of Epidemics-I", Proceedings of the Royal Society, London A, Vol. 115, pp 700-721, 2007.

[17] W. Wang, "Backward bifurcation of an epidemic model with treatment", Mathematical Bioscience, Vol. 201, pp 58-71, 2006.

[18] W. Wang, S. Ruan ,"Bifurcations in an epidemic model with constant removal rate of invectives", Journal of Mathematical Analysis and Application Vol. 291, pp 775-793, 2004.

[19] X. Zhang and X. N. Liu , "Backward bifurcation of an epidemic modelwith saturated treatment function", Journal of

[20] Mathematical Analysis and Applications, Vol. 348, no. 1, pp. 433-443, 2008.

[21] Z. Zhonghua, Yaohong, Suo, "Qualitative analysis of a SIR epidemic model with saturated treatment rate", Journal of Applied Mathematics and Computing, Vol. 34:pp177-194, 2010.

\section{AUTHORS PROFILE}

Ms. Monika Badole passed M.Sc., B.Sc. Mathematics from DAVV, Indore in year $2003 \& 2005$ and She is merit position holder in M.Phil Mathematics from Vikram University, Ujjain in 2011 and pursuing $\mathrm{PhD}$ from Vikram University on Mathematical Modelling. She has more than 8 years of teaching experience and 3

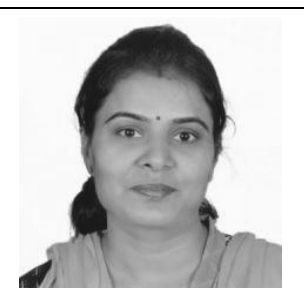
years of research experience. She is currently working as Asst. Professor in Department of Mathematics, Acropolis Institute of Technology \& Research (M.P.) since 2011. Her main research work focused on Mathematical modeling on infectious diseases and trying to develop new idea in the field of Mathematical modeling since 2015.

Dr. Sandeep Kumar Tiwari Prof \& Head School of Studies in Mathematics, Vikram University Ujjain (M.P.) He is working in many field of mathematics research field. He has more than 30 research papers published in national and international peerreviewed reputed journals. He has worked as Asst. Registrar Vikram, University, Ujjain. His area of interest Optimization Techniques, Approximation and Summability Theory, Fuzzy Mathematical Modeling $\mathrm{He}$ has published one book Redefining Business Horizons, MACMILLAN India Ltd. (Advance Research Series, 2011).

Dr. Vandna Gupta passed M.Sc., M Phil, and Ph.D. Mathematics from Vikram University, Ujjain. She has 23 years experience of teaching as Asst. Professor and 11 years experience of teaching as Professor. She is currently working as Professor in Department of Mathematics, Govt. kalidas Girls College Ujjain (M.P.) since 2010. She is life member of Indian Mathematical Society, established

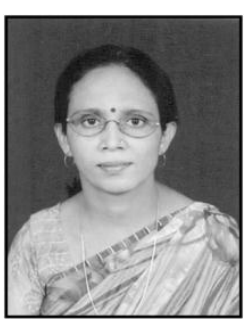
in 1907. Varahmihir institute of Scientific Heritage \& Research Ujjain (M.P.) (Joint Secretary and founder member), an Institute affiliated to MPCST, Bhopal and DST, New Delhi. Vedic Mathematics \& Research, Ujjain. She is member and advisor of Vice Chancellor nominee for R.D.C., Vikram, University, Ujjain (M.P.) and Board of Studies, Vikram University, Ujjain (M.P.). Her area of research is Mathematical Modeling, Fixed Point Theory Approximation Theory. One student has awarded Ph.D. degree under her Supervision from Vikram University and 7 Students currently working for Ph.D. degree in her guidance.

Mr. Ankit Agrawal, formerly an Assistant Professor at the Department of Mathematics, Mahakal Institute of Technology \& Management, Ujjain, is currently an Assistant Professor at the Department of Mathematics, Acropolis Institute of Technology \& Research, Indore. He has more than 6 years of experience in teaching. He is merit position holder in M.Sc. and done M.Phil. from Vikram University, Ujjain and pursuing Ph.D.

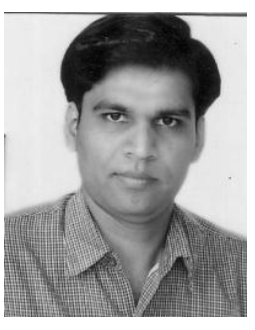
from Barkatullah University, Bhopal. He has also written over 10 research papers 